\title{
Use of bar code readers and programmable keypads to improve the speed and accuracy of manual data entry in the clinical microbiology laboratory: experience of two laboratories
}

\author{
Richard Shaw, John E Coia, Joanne Michie
}

\begin{abstract}
Aim-To assess the effect of the use of bar code readers and programmable keypads for entry of specimen details and results in two microbiology laboratories.

Methods-The solutions selected in each laboratory are described. The benefits resulting from the implementation were measured in two ways. The speed of data entry and error reduction were measured by observation. A questionnaire was completed by users of bar codes.

Results-There were savings in time and in reduced data entry errors. Average time to enter a report by keyboard was 21.1 s $v 14.1$ s for bar coded results entry. There were no observed errors with the bar code readers but 55 errors with keystroke entries. The laboratory staff of all grades found the system fast, easy to use, and less stressful than conventional keyboard entry.

Conclusions-Indirect time savings should accrue from the observed reduction in incorrectly entered data. Any microbiology laboratory seeking to improve the accuracy and efficiency of data entry into their laboratory information systems should consider the adoption of this technology which can be readily interfaced to existing terminals. (f Clin Pathol 1999;52:54-60)
\end{abstract}

Keywords: bar codes; laboratory data; programmable keypads

Unlike in chemistry and haematology laboratories, manual methods are still in widespread use in most clinical microbiology departments. Thus there is limited scope as yet in most microbiology laboratories to download data from analysers onto the laboratory computer system. As a result a great deal of data must still be entered manually by staff. The risk of transcription errors means that manual error checking systems must be an integral part of data entry, which is expensive and time consuming. ${ }^{1}$

The microbiology department at Yorkhill NHS Trust in Glasgow has recently replaced an obsolete laboratory computer system with a new system from CDS TelePath (CDS TelePath Ltd, University of Birmingham Research Park, Birmingham, UK) which calculates the check digits for laboratory numbers using a different algorithm than the previous software.
This meant that we were unable to use check digits on laboratory numbers for the new system during the transition period, when both old and new systems were run in tandem, highlighting the problem of transcription error, as incorrect laboratory numbers were a common occurrence. Accordingly, bar codes were introduced for the laboratory accession number applied to each specimen received in the laboratory. The success of this application prompted us to look further at extending this role, with the perceived advantages of inherent speed and reduced errors of bar codes for entering specimen data and results. Similar requirements existed for the validation of laboratory accession number data and rapid data entry, particularly of "negative" results, into an existing CDS TelePath system in the department of clinical microbiology at the Western General Hospitals NHS Trust in Edinburgh. A very similar system of bar code based data entry was introduced, in this case augmented by programmable keypads.

It was hoped that rapid data entry would release additional technical staff time to carry out the wide range of other tasks essential to the running of a modern NHS diagnostic microbiology laboratory. In addition, by entering results directly onto the computer system using bar codes or keypad entry we anticipated a substantial reduction in errors compared with manual data entry, particularly given the variable keyboard skills mix available among existing staff. We also aimed to speed up the reporting process by decreasing the need to check data.

\section{Methods}

THE DEPARTMENTS

Microbiology Department Yorkhill (MDY)

MDY is a small but busy laboratory providing on-site diagnostic bacteriology and virology services for the paediatric and maternity hospital complex which serves children and pregnant women in Glasgow and provides specialist services for the West of Scotland on the same site. About 75000 specimens are processed each year. Besides a comprehensive range of microbiology services, the laboratory provides the customised examinations required by a highly specialised hospital. The department is accustomed to automation with analysers for blood cultures, antibiotic assays, and enzyme immunoassays and has had a laboratory computer system since 1989 . The original 


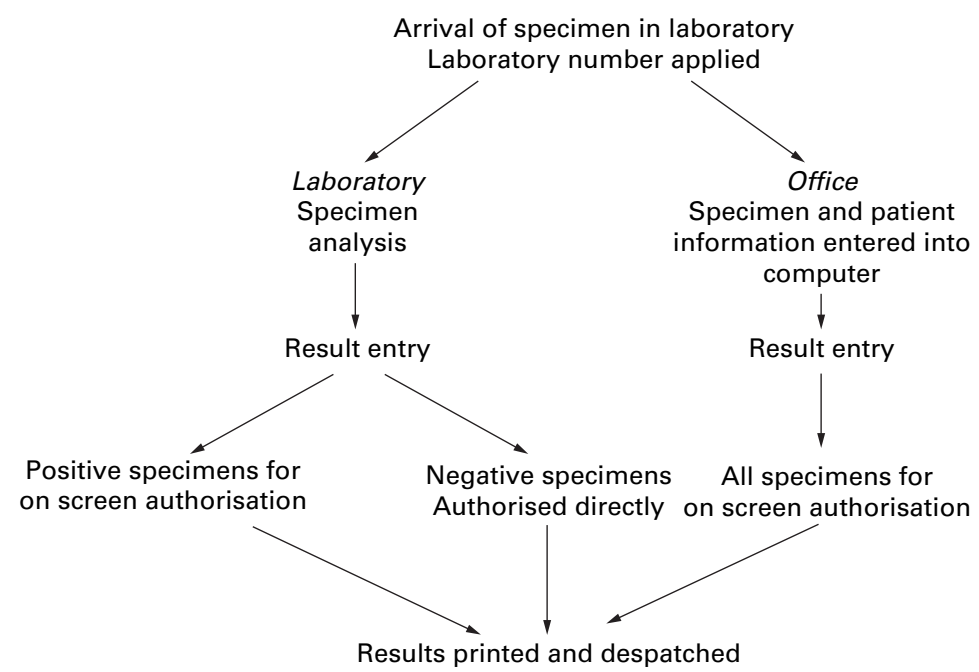

Figure 1 Specimen reception, result entry authorisation, and reporting in the two laboratories.
THE REPORTING PROCESS

Figure 1 shows the processes of receiving specimens, entering results, authorising, and reporting. In both laboratories, all specimen information is entered by clerical staff in the office. Before the introduction of bar codes, the majority of results entry at MDY was also done in the office. However, bar code result entry is so fast that laboratory staff prefer to enter results themselves at the bench, shifting most (DMY) or all (DCM) of the workload over to the left hand side of the diagram. The impact of this change on clerical and technical staff has not been measured. A similar approach has been adopted at DCM as part of an overall strategy to move towards a more "paperless" laboratory environment.

\section{THE HARDWARE}

MDY stores the results of all specimens on a CDS TelePath system which is written in MUMPS and runs on a UNIX platform. The bar code readers are Data Logic DL60 "CCD gun" type scanners which are supplied through a network of distribution agents and are interfaced with Wyse 60 terminals or IBM compatible PCS using CDS TelePath's own product, QTERM, as a terminal emulator. DCM also stores all laboratory results on a CDS TelePath system as outlined above. In this case Altek 6000/65 CCD type bar code scanners were employed either alone or in conjunction with Polytel keyport programmable electronic keypads, which comprise flat rectangular boards on which there were either 176 or 300 user definable touch keys. Keypad programming was achieved by download through a serial link of user definable command files produced in a text editor program on an IBM compatible microcomputer. There is no software limit on the number of keystrokes which may be assigned to a single key, but the on-board EEPROM used to store the definitions has a total capacity of 32000 characters. With the average keypad definition file in our laboratory using 5000 characters, this limit is likely to satisfy even the most demanding applications. These keypads were used in conjunction with replaceable overlays on which the function of each key was indicated. The bar code readers were interfaced either alone or in conjunction with a keypad to existing DEC VT220 terminals.

At MDY, laboratory numbers were printed as bar codes using extended code 39 which allows the full range of ASCII characters mandated by the inclusion of a full stop in the laboratory number. They were printed on specially supplied labels by the biochemistry laboratory at Yorkhill (John McCormick, Buchanan Street, Glasgow, UK). Bar codes for data entry were produced "in house" using LabelRight for Windows and were printed on a laser printer (Worthington Data Solutions, Fenian Street, Dublin, Republic of Ireland). All data entry bar codes used code 128 in order to incorporate carriage returns and control characters into the codes. Code 39 also allows carriage returns but uses space less efficiently than code 128 and does not allow control characters. 

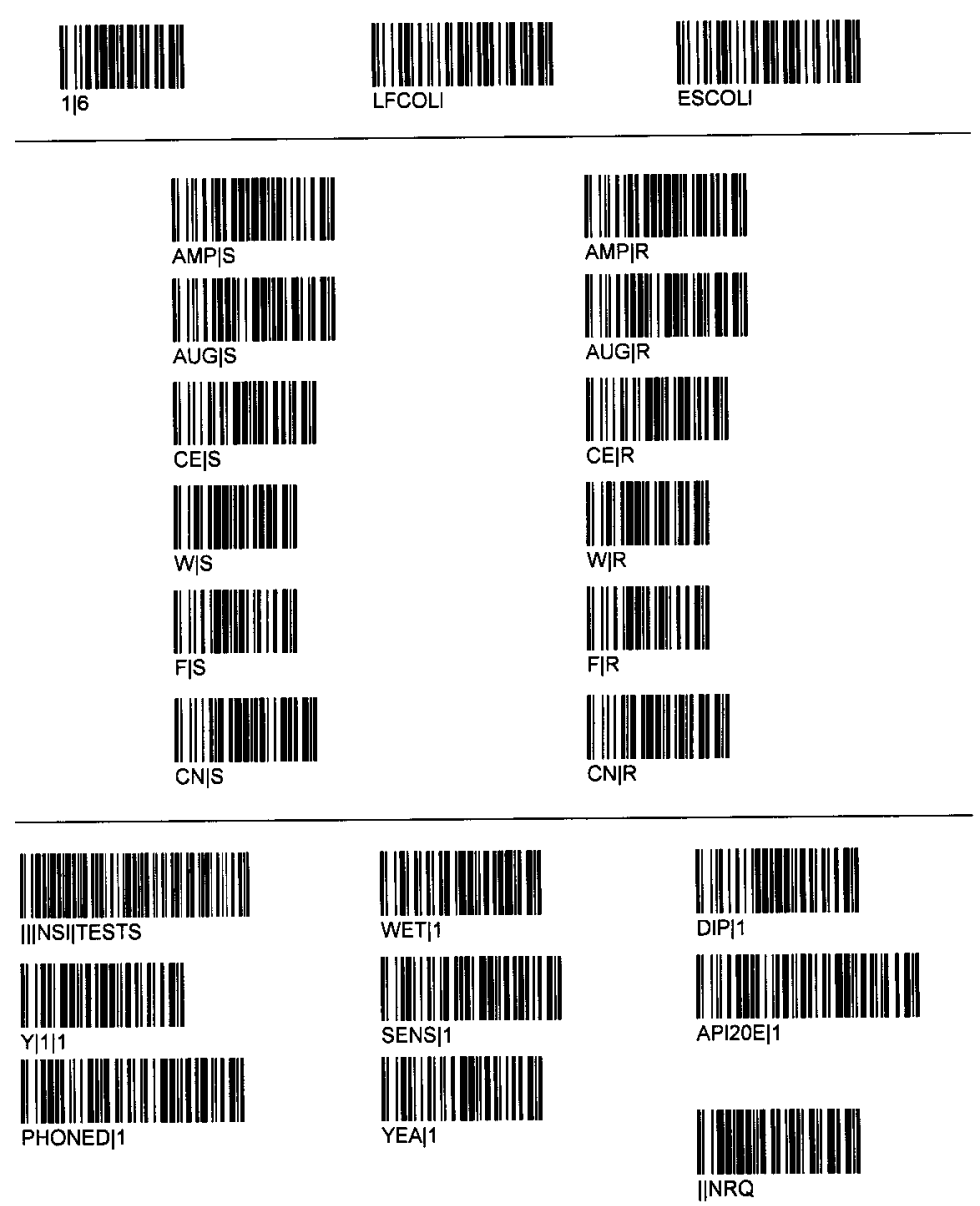

Figure 3 Bar codes for positive urine specimens.

The bar codes were printed on scripts which are sheets of A4 paper which apply to the particular data entry sequence required (figs 2 and 3). Human readable interpretations (HRI) of each bar code are printed on the script. Different scripts are used in highly specific circumstances, for example positive urine results or negative urine results, and can be designed to allow the user to stop at critical stages where different options could be chosen in the same script or where data (such as numerical results) had to be entered at the keyboard. Instructions for their use were included on the script for such occasions.

A similar solution for bar code production was adopted at DCM, although in this case both specimen labels and bar code scripts were produced in house, using LabelRight software in conjunction with a laser printer. Specimen labels incorporated a human readable version of the laboratory accession number which was encoded in code 128 form, as were the codes used in the scripts. The format of scripts was similar to those used in MDY.

\section{IMPLEMENTATION}

In both laboratories, bar coded versions of the laboratory accession numbers were produced on self adhesive labels replacing the conventional laboratory numbers in use at that time. An initial demonstration was set up to train staff in the use of the bar code readers and it rapidly became standard practice to scan the bar code rather than type in the laboratory number on the keyboard.

At MDY, data entry bar codes were first implemented for input of negative blood culture and negative urine results. Crude versions of the scripts were provided which allowed the results of these specimens to be entered. These early versions did not allow carriage returns within the bar code but refined versions very rapidly became available which combined several data entry codes in a single bar code, and carriage returns were included. Within a few weeks scripts were available which applied to about $40 \%$ of all specimen detail entry (for example, specimen type and site sampled) and data entry for $30 \%$ of all reports sent out (predominantly "negative" results entry). Scripts for entry of data in other areas of the laboratory were designed and later versions allowed entry of positive results, including commonly encountered organisms and their sensitivity patterns.

Table 1 Questionnaire on bar codes

This survey is intended to determine how valuable bar code data entry is and how well people adapt to using it. Please answer the following questions by placing a cross in the box provided

1. When entering data how much slower/faster is it when you use bar codes?

$\begin{array}{lll}\text { 2. When using keyboard entry, how often do you need to make corrections? } & \text { Slightly faster } \\ \text { 3. When using bar codes, how often do you need to make corrections? } & & \text { Occasionally } \\ \text { Never } & \square & \square\end{array}$


Table 2 Comparison of error rate and time for report entry

\begin{tabular}{lllllll}
\hline $\begin{array}{l}\text { Entry } \\
\text { method }\end{array}$ & $\begin{array}{l}\text { Number of } \\
\text { specimens }\end{array}$ & Keystrokes & Errors & $\begin{array}{l}\text { Errors/ } \\
\text { keystroke } \\
(\%)\end{array}$ & Time (s) & $\begin{array}{l}\text { Average time per } \\
\text { report (s) }\end{array}$ \\
\hline $\begin{array}{l}\text { Bar code } \\
\text { Keyboard }\end{array}$ & 120 & 0 & 0 & 0.00 & 1692 & 14.1 \\
\hline
\end{tabular}

A very similar roll-out of bar code technology occurred at DCM. However, in this case, although very similar patterns of use were adopted with regard to specimen numbering and specimen detail entry, programmable keypads were selected as the preferred route of result entry, with all staff involved in such work being individually trained at the bench.

MEASURING THE BENEFITS OF RAPID DATA ENTRY TECHNOLOGY

Different aspects of the effects of introducing rapid data entry technology into the laboratory were measured in three ways: (1) speed of data entry; (2) error reduction; (3) user acceptability.

Speed of data entry and error reduction were evaluated by two methods. First, all staff who were required to use bar codes or keypads were asked to complete questionnaires indicating, in terms of a simple scale, how much faster data entry was and how many errors they made using bar code readers or keypads compared with keyboard entry. The questionnaires included questions to determine the acceptability of the bar codes or keypads by assessing the ease of use of rapid data entry technology, its versatility, and how stressful the users found it to be. The responses to the questionnaires allowed a qualitative assessment of the user's perception of the benefits of bar codes for data entry.

The questionnaires were submitted to two clerical and 10 technical staff members at MDY and five clerical and 12 technical staff at DCM, and a $100 \%$ response was achieved. The questions were designed so that all answers were on a scale of 1 (low) to 5 (high) to allow comparison of results (table 1). Responses were received anonymously so no distinction was possible between clerical staff, whose keyboard skills are of a high order, and technical staff, who have widely varying levels of keyboard skills. Since low numbers reduce the statistical validity of the sample, it was considered acceptable to combine staff groups at both sites.

Second, the speed and accuracy of data entry were measured in representative samples of specimens, comparing data entry between bar codes and the keyboard. The specimens were randomly selected and comprised a mixture of positive and negative results. This gave a quantitative estimate of the overall time saved and the rate of keystroke error. A sample of 124

Table 3 Comparison of error rate and time for specimen data entry

\begin{tabular}{lllllll}
\hline $\begin{array}{l}\text { Entry } \\
\text { method }\end{array}$ & $\begin{array}{l}\text { Number of } \\
\text { specimens }\end{array}$ & Keystrokes & Errors & $\begin{array}{l}\text { Errors/ } \\
\text { keystroke } \\
(\%)\end{array}$ & Time (s) & $\begin{array}{l}\text { Average time per } \\
\text { report (s) }\end{array}$ \\
\hline Bar code & 82 & 0 & 0 & 0.00 & 223 & 2.7 \\
Keyboard & 71 & 1640 & 15 & 0.91 & 491 & 6.9 \\
\hline
\end{tabular}

randomly selected reports was entered using the keyboard, and a similar random sample of 120 reports entered by bar code. The time taken to enter each sample was measured and the number of errors counted by observation. The error rate per keystroke was calculated as a percentage. The overall time was divided by the number of specimens in each sample to give the average time (in seconds) to enter the results.

Observation of specimen data entry was done by using a facility in MUMPS ("PEEKing") where the data on screen can be copied to another screen elsewhere and observed remotely, thus reducing the "Hawthorne effect". 2 Data for 82 specimens were entered by bar code and 71 specimens by keyboard. Time taken and error rates were measured in the same way as above.

In addition, the time taken to check the validity of reports before printing was observed. This authorisation process was also observed by "PEEKing". A total of 406 specimens were authorised "on screen" by various members of staff.

\section{Results}

SPEED OF DATA ENTRY

The answers to the questionnaires indicated that staff perceived bar codes and keypads to be faster than keyboard entry. At MDY, all but one reply indicated that staff perceived the bar codes to be very much faster ( 5 on the scale), with the other reply scoring 4 for slightly faster, while at DCM virtually everyone felt that data entry was either much faster (7/17) or slightly faster (7/17).

The average time to enter a report by keyboard was 21.1 seconds. For bar coded results entry this figure was 14.1 seconds (table 2). The range was 9.9 to 15.2 seconds for bar code entry and 14.1 to 40.3 seconds for keyboard entry. Specimen data entry was also faster (table 3).

\section{ERROR REDUCTION}

The respondents to the questionnaires answered questions asking about how often corrections had to be made with keyboard data entry and using bar codes or keypads. The perceived rate of error correction is considerably less with bar codes than with the keyboard with modal replies of "sometimes" for keyboard entry and "rarely" for bar code entry at MDY, and "occasionally" for keyboard entry and "rarely" for bar code or keypad entry at DCM.

Measurement showed no observed errors with the bar code readers but 55 errors, representing $0.83 \%$ of all keystrokes, occurred. Detailed analysis of the sample shows that for some specimens this error rate was as high as $3.9 \%$. Errors were observed in an estimated $25 \%$ of all reports entered using the keyboard although this was not accurately measured.

\section{ACCEPTABILITY}

All respondents to the questionnaires found bar codes to be superior in ease of use to keyboard entry. All but two respondents at MDY stated that bar codes were "much easier" to 
use; the other replies indicated they were "slightly easier" to use. At DCM almost all respondents felt bar codes or keypads were either "much easier" (8/17) or "slightly easier" (647) to use than keyboards.

Seven respondents at MDY replied that bar code readers were compatible with, and fitted very well into, the process of reporting, but three replies stated that they fitted quite well and two others that they were about the same. At DCM the results were broadly similar with most $(10 / 17)$ of the opinion that bar code readers or keypads fitted "very well," four that they fitted "well," and three who felt they were "about the same."

The respondents to the questionnaires at MDY all expressed a clear preference for using bar codes when available, with the modal answer being used "whenever possible" (eight respondents), and four respondents replied "much of the time." Again the DCM results were similar, with $11 / 17$ stating that they used bar codes or keypads "whenever possible" and $4 / 17$ "much of the time". The remaining two respondents used either method "half of the time."

All respondents at MDY reported that data entry was "not stressful" with bar codes, and four respondents reported the same for keyboard entry. Eight respondents found keyboard entry to be "slightly stressful." Over half of the DCM respondents (10/17) found keyboard data entry stressful ( 6 slightly; 3 moderately; 1 highly), while only $6 / 17$ felt any stress in using bar codes or keypads (all six at the "slightly" level).

\section{AUTHORISATION}

The average time taken to authorise a specimen was 11.2 seconds. The minimum time taken was 7 seconds and the maximum time $63 \mathrm{sec}-$ onds. Of the reports, $90.6 \%$ were authorised in an average time of 10.2 seconds with a minimum of 9 and a maximum of 12 seconds.

\section{Discussion}

Bar code and keypad technology are in widespread use in a variety of industrial and retail settings, particularly in stocktaking and electronic point of sale (EPOS) applications. In the clinical laboratory this technology has most commonly been used in specimen numbering and identification applications. All diagnostic NHS laboratories are under continuing pressure to carry out more analyses on increasing numbers of specimens. Resources are limited and therefore it has become necessary to find ways to minimise the time spent on "clerical" activities such as specimen data entry and error correction. In response to this pressure, we attempted to speed up the process of specimen and result entry into our microbiology computer systems by adopting these rapid data entry technologies.

There are few reported examples of the use of bar codes in microbiology laboratories for result entry, and most of these come from the USA. ${ }^{34}$ However, reports from various disciplines indicate the benefits of bar code technology.
Specimen identification using bar code readable labels is widely used in clinical laboratories and has proven valuable in reducing errors, improving the service, and producing labour savings. ${ }^{5-7}$ Bar codes in other disciplines have primarily been used in specimen and product management by identifying the specimen as it passes through the laboratory. ${ }^{3}$ However, in at least one cytology laboratory, keyboard entry of results has been made all but obsolete. ${ }^{8}$

By applying the proven technology of bar codes, we expected the same advantages of error reduction and greater speed reported elsewhere. ${ }^{349}$ Measurement of data entry confirmed that we have achieved an increase in speed, and the process is so fast that laboratory staff feel inclined to enter the data themselves whereas previously they had handed reports to clerical staff for transcription onto the laboratory computer. Not only is result entry faster but another possible source of error is removed and this drastically reduces the need to check that the reports have been transcribed correctly, leading to further time savings. For example, at MDY the time taken for data entry has been reduced by an average of 7 seconds per report where bar codes have been applied, affecting some 48000 reports with an estimated saving of 336000 seconds or around 93 hours a year.

Responses to the questionnaires indicate that errors in data entry are less common with bar codes than with keyboard entry. This perception is supported by the observation that, for both reports and specimen data entry, there were no observed errors with the bar code readers. This does not suggest that errors are eliminated but that our observation period was too short to observe any. While the error rate with keyboard entry was less than $1 \%$ for both reports and specimen data, it was clear that enough errors occurred to contribute to the increased time for data entry. Other reports indicate that the error rate in bar code use is very low. ${ }^{8}$ The reduction in errors allows the release of many specimens for printing without further checking, an additional saving of more than 10 seconds per report.

Errors have not been eliminated and it remains possible for people to enter wrong reports, such as "No growth" instead of "No significant growth." However, the use of bar codes greatly restricts the opportunity for incorrect reports and both laboratories have made considerable use of technical validation in TelePath to prevent the reporting of incorrect results. The validation facility may not be available or as highly developed in other computer systems and reducing the options for data entry by using limited bar codes can in itself help to reduce errors.

The range of bar code scripts in use at MDY is still under development and we now have around 40 available. Only 12 of the result entry scripts are in regular use and a similar number are used for specimen entry. These account for about $70 \%$ of specimens and $60 \%$ of reports. The majority of reports require a mixture of bar code and keyboard entry, for example where dates or numerical data are entered, but 
this has been minimised where possible. In principle, it is possible to provide bar codes for numbers or dates but this may be impractical because of the large number of bar codes required to account for the very wide variation of numerical data.

At DCM, keypads have now been implemented as the standard method of result entry in all areas except public health (which will follow in due course). The system has proved popular with all grades of staff, and greatly simplifies the use of the underlying "core" software. The use of the keypads has benefits in limiting the number of switches between different input devices required, and does not require a large number of separate scripts to be held at terminals.

At the time of writing MDY are developing a process where checking of reports will be eliminated for many specimens, especially those which are negative. The restricted availability of bar codes combined with the use of validation rules built into the TelePath system will make it very difficult to accidentally release an incorrect report for printing. This may eventually be applied to as many as 30000 reports with an estimated saving of 300000 seconds ( 83 hours). We predict, therefore, that we will save as much as 176 whole time equivalent (WTE) hours a year as a direct result of our bar code implementation. This does not take account of any time savings as a result of reducing errors in those reports which will still require error correction or other use of bar codes in future.

Ready acceptance of bar code and keypad technology among those who use it is in contrast to the adoption of the computer system itself. Before the introduction of rapid data entry technology users found data entry to be somewhat cumbersome and it took some time before they became proficient with unfamiliar codes. Although the introduction of the new data entry hardware caused some initial problems as staff became used to operating the readers and keypads, after a very short time they became very adept.

It was notable that in the questionnaire returns none of the respondents replied that keyboard entry was a better option. Although no distinction was made between the levels of keyboard skills possessed by respondents to the questionnaires, none of the responses indicates a negative attitude and all respondents seem to view the bar codes in a favourable light. It is worth remembering, however, that the bar codes replace keyboard skills to a large extent and these skills are the territory of clerical staff whose initial reluctance to use bar codes may stem from a fear of being replaced by the technology. This may be especially the case at MDY where much of the work previously done by clerical staff is now done at the bench.

One of the reasons why bar code and keypad technology have proven popular is because they remove the need to remember codes. Individuals unfamiliar with the reporting of particular specimens need only use the appropriate bar codes or keypad press. Interestingly, even users who would have been familiar with the system of reporting became confused with the complexity of the computer when entering results through the keyboard. In contrast, all of those who use bar codes or keypads find them easy and speedy in use. There is no evidence that users find rapid data entry technology difficult to adopt as part of their daily routine, and many users have readily suggested modifications or additions to the repertoire of items which can be entered in this way. The level of demand for these additions is testament to the popularity of these novel modes of data entry.

While most terminals or terminal emulators allow programming of a small number of function keys to enter particular keystroke sequences, even these are limited in terms of the number of keystrokes which can be encoded on a single key. Any sequence of keystrokes can be entered by bar code or keypad. Some common organisms with predictable sensitivity patterns are entered by this method as well as frequently used comment codes. If function keys on standard terminals are programmed the programming applies only to the keys on that particular terminal and this is not easily transferable to another terminal. Bar codes can be carried to another site and used there without modification, and the keystroke definition files for dedicated programmable keypads are easily downloadable to new keypads, or those at other sites. Standard terminal function keys perform their tasks blind unless a "menu" of programmed keys is available on the keyboard. These menus can become cluttered or lost. In contrast, the bar code scripts have a human readable version printed next to the bar code so that the user can read the sequence of keystrokes about to be initiated, and dedicated keypads have user customisable overlays to readily permit the user to see what the key will do.

Speech controlled data entry has been used in some laboratories. ${ }^{10}$ This technology is promising but at present there is still a high local processing overhead at the terminal requiring expensive hardware for each individual user. In addition these systems currently require considerable input to "train" the software to understand the user's voice. The rapid data entry systems we have described here are available immediately and can be used by anyone with a minimum of training. In addition the hardware is inexpensive and is virtually "transparent" to the existing hardware, permitting simple interfacing to existing low cost character based terminals or PCs.

The introduction of rapid data entry technology has had a major effect in our laboratories. Bar code data entry at the laboratory bench is seen as a crucial part of the strategy which aims to create a "paperless" laboratory environment. Meanwhile, at MDY, changes in working practices have made it possible to send out printed reports up to six hours earlier than was previously possible with potential benefits to patient care. Yorkhill NHS Trust will acquire an HISS in the near future. This will allow reports to become available on ward terminals as soon as they are authorised, and patient care may directly benefit from the speed of report 
entry. It is hoped that a large proportion of reports will be available for enquiry on ward terminals before 10.00 am on the day after they are received. We also hope that this will lead to a reduction in the number of telephone inquiries to the department.

The anticipated benefits of introducing bar code technology have been realised in our laboratories. For example at MDY measurable time saved is the equivalent of employing 1 WTE for more than four weeks and, at the same time, considerable error reduction has been achieved, improving the quality of the laboratory output. These advantages have been gained without any discomfiture to the laboratory staff, who perceive greater productivity without greater effort from themselves. The psychological importance of this should not be underestimated. Maddix stated that many people sitting in front of a computer would rather be doing something else. We believe they would be satisfied just doing it better. ${ }^{11}$

1 Tilzer LL, Jones RW. Use of bar code labels on collection tubes for specimen management in the clinical laboratory. Arch Pathol Lab Med 1988;112:1200-2.

2 Kanter RM. The change masters. London: Routledge. 1984:409.

3 Willard KE, Shanholtzer CJ. User interface reengineeringinnovative applications of bar coding in a clinical microbiology laboratory. Arch Pathol Lab Med 1995;119:706-12.
Aller RD, Friedman W. Rapid accurate entry of microbiolAller RD, Friedman W. Rapid accurate entry of micro
ogy results. Arch Pathol Lab Med 1996;120:57-61.

5 Dito WE, McIntire S, Leano J. Bar codes and the clinical laboratory: adaption perspectives. Clin Lab Management Rev 1002;6:72-85.

6 Smith JE, Meyer, GE. Organizational approach to implementing bar code technology in a university hospital. Am $\mathcal{F}$ Hosp Pharm 1987;44:572-3.

7 Neeley WE. design of a bar code identification system for the clinical laboratory. Informatics Pathol 1987;2:159-62.

8 Aller RD, DeWitt C. Cytology result entry without using the keyboard. Acta Cytol 1994;38:739-41.

9 Petts D, Meacock T. Use of bar codes in microbiology com-

puterization. Microbiol Europe 1996;4:12-18.
10 O'Hara SP, Athersuch R. Speech recognition and direct data entry in clinical microbiology. $\mathrm{Br} \mathcal{F}$ Biomed Sci

11 Maddix F. Human computer interaction: theory and practice. London: Ellis Horwood, 1990:256. 